\title{
Value of Teledermoscopy in Primary Healthcare Centers: Preliminary Results of the TELESPOT Project in Belgium
}

\author{
Thomas Damsin • Gregory Canivet - Pauline Jacquemin • \\ Laurence Seidel · Pierre Gillet • Didier Giet · Arjen F. Nikkels $\mathbb{B}$
}

Received: August 13, 2020 / Published online: September 18, 2020

(C) The Author(s) 2020

\begin{abstract}
Introduction: Teledermoscopy using smartphone-based applications is becoming more and more important in a setting of increasing frequency of skin cancer and difficult access to specialized care. The TELESPOT project aimed to provide rapid diagnosis and speed up patient
\end{abstract}

Digital Features To view digital features for this article go to https://doi.org/10.6084/m9.figshare.12906749.

T. Damsin · A. F. Nikkels ( $\square)$

Department of Dermatology, University Hospital

Centre, CHU du Sart-Tilman, Liege, Belgium

e-mail: af.nikkels@chu.ulg.ac.be

G. Canivet

Department of Computer Applications, University

Hospital Centre, CHU du Sart-Tilman, Liege,

Belgium

P. Jacquemin

Department of Methods of Projects, University

Hospital Centre, CHU du Sart-Tilman, Liege,

Belgium

L. Seidel

Department of Biostatistics, University Hospital

Centre, CHU du Sart-Tilman, Liege, Belgium

P. Gillet

Medical Director, University Hospital Centre, CHU

du Sart-Tilman, Liege, Belgium

D. Giet

Department of General Medicine, University

Hospital Centre, CHU du Sart-Tilman, Liege,

Belgium flow between primary healthcare centers and a tertiary care center in Belgium. The aim of the present study is to describe the development of an in-house smartphone-based dermoscopy application, evaluate its real-life value in a series of primary healthcare centers, and present preliminary diagnostic data.

Methods: Modified Likert scales were used to assess patient and general practitioner (GP) satisfaction rates for the system. Furthermore, a total of 105 photographic and dermoscopic images were acquired in a series of 80 patients at participating centers.

Results: Overall, patient and GP satisfaction levels were $89 \%$ and $94 \%$, respectively. Highpriority management was recommended in 7.6\% of cases (8/105: 3 basal cell carcinoma, 1 primary cutaneous B-cell lymphoma, 1 Spitz melanocytic nevus, 1 congenital nevus, 1 in situ melanoma, and 1 invasive melanoma, proven by histology).

Conclusions: The primary healthcare centers were highly satisfied with the TELESPOT project in terms of user-friendliness, efficacy, and reliability as well as in providing a reinforced image of first-line medicine efforts in combating skin cancer.

Keywords: Melanoma; Primary healthcare; Public health; Skin cancer; Teledermatology; Teledermoscopy 


\section{Key Summary Points}

The TELESPOT project is based on a teledermoscopy device and smartphone application to be used for skin cancer detection in primary healthcare centers.

Both physicians and patients participating in the TELESPOT teledermoscopy project reported very high satisfaction levels.

In terms of skin cancer management in primary healthcare centers, teledermoscopy is a useful tool to preselect patients and allows rapid access to specialized care.

\section{DIGITAL FEATURES}

This article is published with digital features to facilitate its understanding. To view digital features for this article go to https://doi.org/10. 6084/m9.figshare.12906749.

\section{INTRODUCTION}

The incidence of skin cancer, including basal cell carcinoma (BCC), cutaneous squamous cell carcinoma (cSCC), melanoma, and Merkel cell carcinoma, has been steadily increasing for decades $[1,2]$. This evolution has led to a higher workload for general practitioners (GPs) and dermatologists. Preselection of patients could avoid unnecessary specialist visits. In addition, insufficient awareness from the general population and care providers at primary healthcare centers (PHCs), distance to specialized diagnostic centers, and long waiting lists for appointments are other factors that hamper early diagnosis $[3,4]$. In terms of public health, early diagnosis remains the cornerstone of reducing skin cancer morbidity and mortality [5].

Although dermoscopy has steadily increased the diagnostic accuracy of skin cancer, its use is limited in first-line medicine [6, 7]. Teledermoscopy (TDS) allows image analysis at a distance. This is less operator dependent compared with teledermatology, as acquisition of images is standardized and highly reproducible [8]. The sensitivity and specificity of TDS have been established previously [9].

This background initiated the TELEdermoscopy Smartphone-based Pigmented lesion diagnosis Online Taskforce (TELESPOT) project [8]. Participating PHCs were trained to acquire macroscopic and dermoscopic images for every lesion judged as suspect with a brief description of patient data. Images were transmitted securely to a tertiary center for analysis by two experienced dermatologists. After analysis, a brief report was sent back within 5 working days to the GP with advice for patient management and diagnosis if possible (Fig. 1).

This article describes the TELESPOT project and aims to assess patient and GP satisfaction levels. Furthermore, preliminary data on management advice and diagnostic results are presented.

\section{METHODS}

This study was performed in accordance with the Helsinki convention on human rights. The ethics committee of CHU du Sart-Tilman and the university hospital legal department approved the project. Patients were informed about all procedures, and they all signed informed consent forms.

\section{Smartphone Application Development}

Each participant GP is logged in through a portal authenticator with a unique login and password. The digital identifier can also be used on a smartphone with a mobile number and the Itsme $^{\circledR}$ application, which generates a unique combination guaranteeing secure access to personal data.

Data are uploaded onto the Orthanc ${ }^{\circledR}$ platform, a lightweight open-source digital imaging and communications in medicine (DICOM) server for medical imaging supporting application programming interface representational 


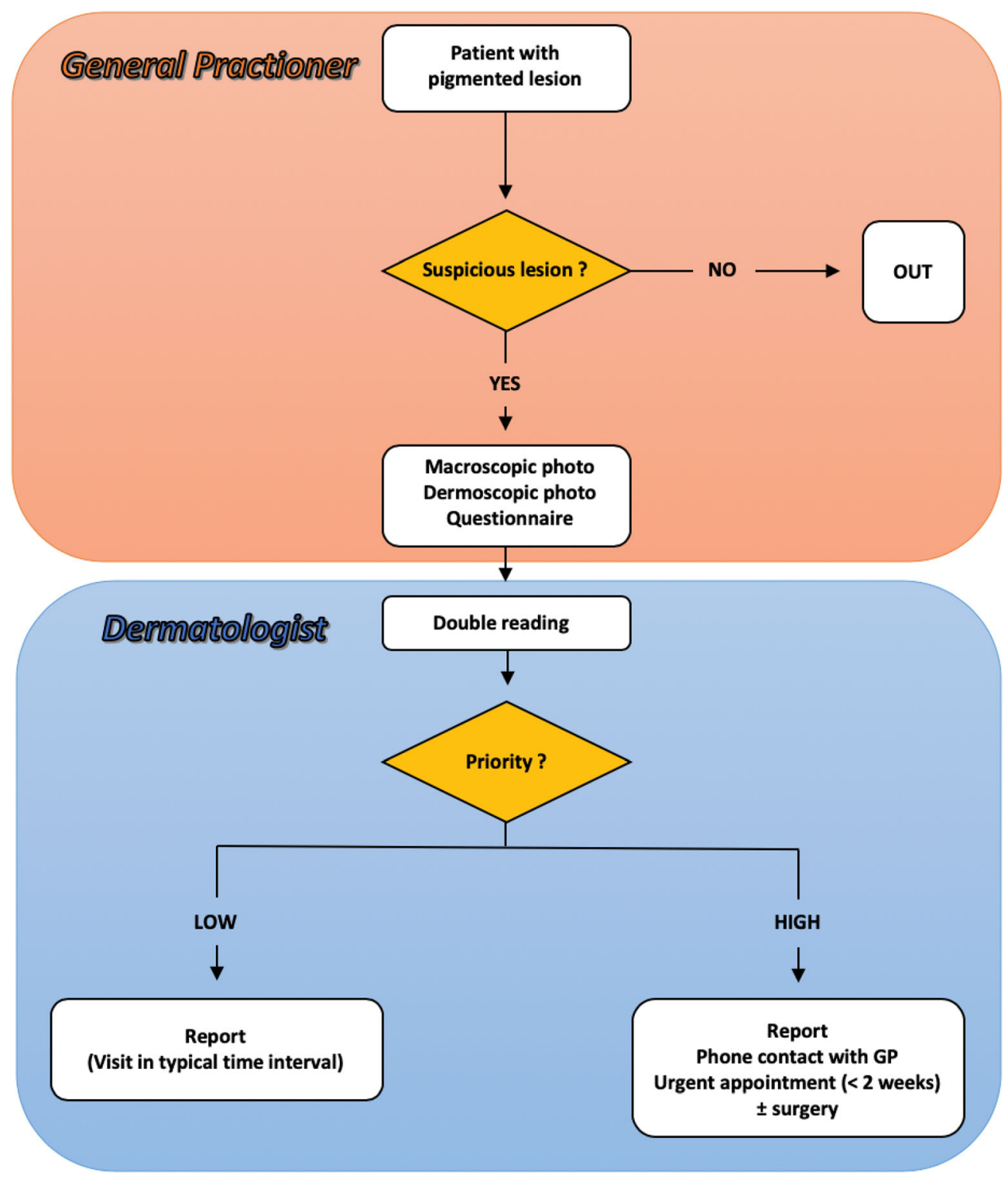

Fig. 1 Workflow in TELESPOT project

state transfer ( REST) providing interoperability between different computer systems.

The acquired dermoscopic pictures are converted into digital slides. Using whole-slide imaging (WSI), clinical and macroscopic pictures are scanned, and a single high-resolution digital file is created. This is commonly achieved by capturing many small high-resolution image tiles or strips and then converting these into a full image. This conversion accelerates data transfer and visualization of the images. Only the visible files are loaded. The web server is an Apache HTTP server, a free and open-source cross-platform web server software. Patient administrative data are transferred to the hospital information system Oazis ${ }^{\circledR}$. Clinical data are transferred to the electronic patient record (EPR), where they are analyzed and feedback is generated. Images are also stored into the picture archiving and communication system (PACS) for long-term archiving and internal diffusion with EPR. Data processing is shown in Fig. 2. 


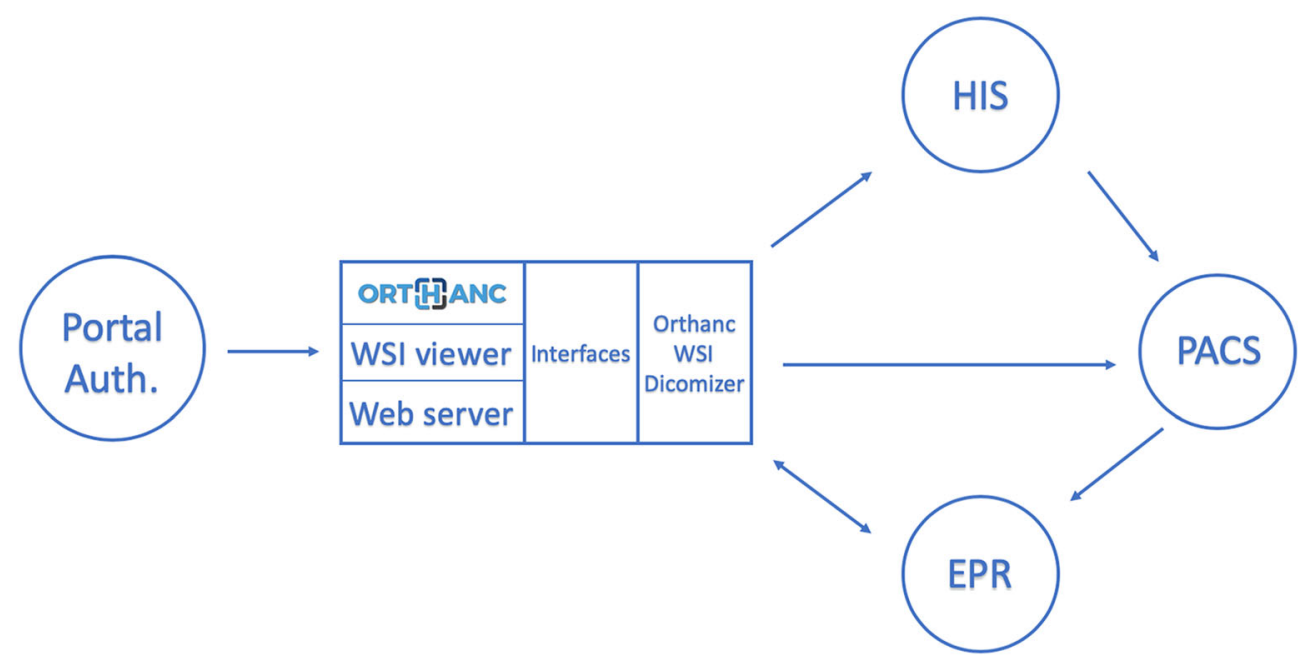

Fig. 2 Data processing in TELESPOT system

\section{Smartphone Dermoscopic Device}

Each PHC is equipped with a smartphone (iPod ${ }^{\circledR}$ Touch 7; Apple, Cupertino, CA) and a handheld dermatoscope compatible with the smartphone (Heine ${ }^{\circledR}$ ic1; Heine Optotechnik, Herrsching, Germany).

\section{Training of PHC and Visits}

Each PHC received a digital presentation $\left(\right.$ Prezi $^{\circledR}$ software; Prezi ${ }^{\circledR}$ Inc., Budapest, Hungary) with three topics-introduction on pigmented skin lesions, essentials of dermoscopy, and operation of TELESPOT project-a dermoscopy quiz, and an on-site training with the device and application. A second visit was organized after the first cases to improve and/or rectify the quality of acquisitions. Other on-site visits were at PHC or investigator request.

\section{Project Settings}

Six PHCs were selected within a $15-\mathrm{km}$ radius of the tertiary center. All PHCs work with a multidisciplinary team, totaling 42 GPs (mean age: 44 years), 22 nurses, and other paramedical/administrative staff.

\section{Screening Items}

The following items were assessed: quality of acquisition, evolution of lesion over time, nature of lesion, diagnosis, management priority with specific management recommendations according to the proposed TELESPOT diagnosis (Table 1), histopathology of high-priority lesions, time to face-to-face (FTF) visit, and/or surgery for high-priority lesions.

\section{Satisfaction Scores}

A modified Likert scale was used to record physician satisfaction score $(n=6)$ by telephone visits, assessing 10 items with a score from 0 to 10. Patient satisfaction $(n=19)$ was assessed by telephone visits using the same method, with a total of five items and three binary questions.

\section{Statistics}

Instead of performing a power calculation, given the limited number of cases, a 95\% confidence interval for the proportion of high-priority lesions was determined. This confidence interval goes beyond just providing the observed proportion by giving a range of potential values for the proportion studied; it is associated with the observed proportion of high-priority lesions, namely $7.6 \% \quad(8 / 105$; 
Table 1 Distribution of diagnoses on TELESPOT acquired images

\begin{tabular}{llll}
\hline Diagnostic category & $n=105(\%)$ & \multicolumn{2}{l}{ Specific management } \\
\cline { 4 - 4 } & & $\begin{array}{l}\text { Priority } \\
\text { recommendation }\end{array}$ & $\begin{array}{l}\text { Treatment } \\
\text { recommendation }\end{array}$ \\
\hline Actinic keratosis & $5(4.8 \%)$ & Low & $\begin{array}{c}\text { Cryotherapy or other } \\
\text { destructive therapy }\end{array}$ \\
Angioma & & & Abstention \\
Atypical nevus & $4(3.8 \%)$ & Low & Closed follow-up \\
Basal cell carcinoma & $4(3.8 \%)$ & Low & Surgery \\
Benign nevus & $3(2.8 \%)$ & High & Follow-up \\
Dermatofibroma & $46(43.8 \%)$ & Low & Abstention \\
Keratoacanthoma & $3(2.8 \%)$ & Low & Abstention \\
Lentigo simplex & $1(1 \%)$ & Low & Follow-up \\
$\begin{array}{l}\text { Melanocytic lesion highly suspected of malignancy } \\
\text { Other benign lesions (macrocomedo, acanthoma, }\end{array}$ & $4(3.8 \%)$ & High & Surgery \\
chondrodermatitis nodularis helicis, & $4(3.8 \%)$ & Low & According to diagnosis \\
and pyogenic granuloma) & & & Low \\
Seborrheic keratosis & & & Low \\
$\begin{array}{l}\text { Other malignant lesions } \\
\text { (primary cutaneous B lymphoma) }\end{array}$ & $23(21.9 \%)$ & High & Abstention \\
\hline
\end{tabular}

range $1.0-11.8 \%$ ). Thus, the observed proportion differs from the true one by $5 \%$ with a confidence of $95 \%$. Should a lower difference be required, i.e., 3\%, at least 300 lesions would have to be included.

\section{RESULTS}

\section{Satisfaction Scores}

Using a set of 10 questions, physician satisfaction scores were the following: (1) the project easily fits into daily practice: $8.6 / 10$; (2) the acquisition technique is not very time-consuming: 9.4 ; (3) satisfaction with the report and advice: 9.6 ; (4) the project accelerates diagnosis of suspicious skin lesions in my patients: 9 ; (5) the project represents a health benefit for my patients: 8.8 ; (6) involvement in skin cancer screening: 8.6 ; (7) improving diagnostic competences in distinguishing benign versus malignant skin lesions: 6.8 ; $(8)$ more eager to do a complete skin check-up: 7.6; (9) the project adds value to PHC: 9.2; and (10) general satisfaction with the project: 9.4.

Patient satisfaction results, assessed using five questions, were the following: (1) comfort with procedure: $9.4 ;(2)$ confidence about this new technology: 8.6; (3) trust in specialized advice: 8.2 ; (4) willingness to repeat the experience: 8.8; and (5) general satisfaction with the project: 8.8. Three subsidiary questions were assessed with a binary answer: (1) did you consult your GP for this specific lesion or was it your GP who proposed the analysis? 16\%: GP, 84\%: patient; (2) did you seek an in-person appointment with a dermatologist to analyze this lesion? 42\%: no, 58\%: yes; and (3) did you have an examination of this lesion by a dermatologist after the TDS project? 16\%: yes; 84\%: no. 


\section{Screening Results}

A total of 105 lesions were analyzed. Among the 80 patients, $67.5 \%$ were female. The mean age was 48.5 years (6-89 years). A total of three acquisitions were judged as impossible to assess and were asked to be repeated. According to patients, 49/105 lesions were present $>1$ year and $6 / 105<3$ months. Following double-blinded assessment of clinical data and clinical and dermoscopic images, $86.7 \%$ of lesions were classified as benign, $5.7 \%$ as uncertain, and $7.6 \%$ as malignant (Table 1 ). Specific management was issued for every single case. For $92.4 \%$ of lesions, low-priority management was advised. High-priority advise was given for three basal cell carcinoma, one primary cutaneous B-cell lymphoma, one Spitz melanocytic nevus, one congenital nevus, one in situ melanoma, and one invasive melanoma. All clinical diagnoses were histopathologically confirmed. The median delay between TELEPSOT diagnosis and treatment for high-priority lesions was 11 days.

\section{DISCUSSION}

In contrast with the smartphone-based TDS referral system of Börve (iDoc24 $\mathrm{PRO}^{\circledR}$; iDoc24 Inc., Berkeley, CA) [10] and the Handyscope ${ }^{\circledR}$ application and FotoFinder $\mathrm{Hub}^{\circledR}$ system (FotoFinder Systems GmbH, Bad Birnbach, Germany) used by Vestergaard [11], the development of our system was based on opensource applications and programs. The main advantages of open-source development are flexibility, sustainability, security, and reduced costs [12].

The first system [10] and our application are principally focused on distinguishing benign versus malignant lesions and on prioritizing clinical management. The impact on skin cancer care of our TDS referral system was highly appreciated in all PHCs, in good agreement with results from previous reports [11, 13]. Patient satisfaction was not assessed in those studies.

As very high sensitivity and specificity rates of the TDS system have already been published, our study did not include an FTF control. In fact, no significant difference in sensitivity was observed between FTF consultations and TDS referrals, especially concerning distinguishing benign versus malignant: diagnostic accuracy for primary diagnosis and benign versus malignant triage with TDS were $58.2 \%$ (95\% CI 52.3-63.9) and $80.1 \%$ (95\% CI 75.0-84.5), respectively [9]. In detail, this situation reflects better the final aims of TDS in real-life healthcare conditions: downsizing unnecessary FTF visits and accelerating management of suspicious lesions.

Our demographics are comparable to two other studies: female: $61.4 \%$, mean age: 54 years; female: 63\%, mean age: 56 years $[10,11]$.

Only $2.9 \%$ of the acquired images were discarded (low picture quality, out of focus images, or missing macroscopic or dermoscopic images). This value is intermediate regarding the other two studies, reporting $0.4 \%$ [10] and 9.5\% [11]. This confirms the high reproducibility of image acquisition and underlines the reliability of the system, hence excluding an important bias observed in general teledermatology image acquisition and picture quality.

A total $86.7 \%$ of lesions were classified as benign and $92.4 \%$ as low priority. Melanoma represented $1.9 \%$ of all lesions and $25 \%$ of highpriority lesions. Another study classified $71.9 \%$ of lesions as benign versus $28.1 \%$ as premalignant/malignant. Melanoma was detected in $4.3 \%$, and low priority was given in $83.8 \%$ [10]. Another study scored $72.3 \%$ as benign lesions versus $27.7 \%$ as premalignant/malignant lesions, including 3.8\% scored as melanocytic malignant lesions [11]. The last study did not provide advice in terms of management priority.

For high-priority lesions, an FTF visit was scheduled for the week following the sorting report, and if necessary, surgery was performed immediately. Management of a high-priority lesion was seven times faster in comparison with the conventional care pathway (median waiting time for an FTF visit: 84 days). In Börve's study, this factor was reduced by three [10]. This difference is possibly explained by the preexisting triage with traditional paper 
referrals in Sweden in contrast to Belgium, where direct access to specialized care is possible.

A worrying result was that $46.7 \%$ of acquired lesions were present for more than 12 months and only $20 \%$ were present less than 3 months. No comparable data are available from the other studies $[10,11]$. This indicates that individuals are still not aware of the risks of skin cancer and that the sooner the diagnosis is made, the better the prognosis is. This stresses once again that repetitions of skin cancer detection programs and awareness campaigns still remain mandatory [14].

In contrast with the initial aim of providing a diagnostic aid for pigmented lesions, a large number of lesions turned out to be nonpigmented, suggesting a need for diagnostic help at PHC's not only for pigmented lesions.

The high global satisfaction score of 9.4/10 indicates that TELESPOT was easily integrated at PHCs. Acquisitions were usually performed by one dedicated GP or nurse. The technique was judged not very time-consuming and considered as a real healthcare benefit for patients. In addition, GPs felt more active in the fight against skin cancers, although they still tend to refrain from performing a total body examination. Lack of time could be an explanation.

The GP's positive predictive value for malignant/premalignant lesions in general was $49.5 \%$, and $26.3 \%$ for melanoma [11]. Indeed, $73.7 \%$ of GPs felt unconfident proposing a diagnosis of melanoma [11]. Although this study did not directly evaluate this issue, it was indirectly reflected in the $6.8 / 10$ score for the feedback question "improving diagnostic competences in distinguishing benign versus malignant skin lesions." Moreover, a Belgian study evaluated diagnostic ability of GPs and dermatologists in discriminating pigmented skin lesions: GPs' versus dermatologists positive predictive values in discriminating malignant from benign disease were $61 \%$ and $92 \%$, respectively [15]. Their respective sensitivity and sensibility percentages were $72 \%$ versus 91\% and $71 \%$ versus 95\% [15]. Another study revealed that GPs' sensitivity and specificity in regards to any malignancy/premalignancy was
$87.8 \%$ and $59.6 \%$, respectively, and for melanoma, $52.6 \%$ and $93.6 \%$, respectively [11].

Evaluated at 6 months, the TELESPOT project seems to constitute a helpful tool at PHCs for early skin cancer detection, but did not increase willingness to be more involved personally. This outsourcing of a task to a third party was previously observed by other TDS referral systems. Another study found that $97.4 \%$ of TDS referrals were reported as helpful by GPs [13].

This type of project could be helpful as diagnostic aid in many settings, such as improving dermatological care in developing countries [4].

Finally, patients were highly satisfied with the TELESPOT project (global satisfaction: 8.8/ $10)$, although use of telemedicine and teledermatology is not yet part of their daily life in Belgium. Our results are in line with other studies reporting patient satisfaction levels of $58.5 \%$ [16].

One limitation to our system (and to other TDS systems) remains the initial triage deciding whether a lesion is to be analyzed or not, which may miss rare clinical presentations such as amelanotic melanoma [7]. However, the classification of more than $90 \%$ of acquisitions as low-priority management indirectly indicates the possibility of an effective screening at PHCs. In addition, the fact that only 7/105 lesions were sent with a prediagnosis is another indicator that outsourcing of this diagnostic act is preferred.

\section{CONCLUSIONS}

This preliminary report on the TELESPOT project confirmed its general usefulness, easy implementation, and user-friendliness. Both PHCs and patients judged the TELEPSOT system as highly beneficial for improved quality of healthcare. Speed of management of suspicious lesions was increased sevenfold by reducing unnecessary FTF visits. Long-term evaluation will determine its final place in the fight against skin cancer. 


\section{ACKNOWLEDGEMENTS}

We would like to thank you all the patient and general practitioner participants who taking time for the acquisition and feedback.

Funding. No funding or sponsorship was received for this study or publication of this article. The journal's Rapid Service Fee was funded the Department of Dermatology, CHU of Sart Tilman.

Authorship. All named authors meet the International Committee of Medical Journal Editors (ICMJE) criteria for authorship for this article, take responsibility for the integrity of the work as a whole, and have given their approval for this version to be published.

Disclosures. Thomas Damsin, Gregory Canivet, Pauline Jacquemin, Laurence Seidel, Pierre Gillet and Didier Giet have nothing to disclose. Arjen F. Nikkels is a member of the journal's Editorial Board.

Compliance with Ethics Guidelines. This study was performed in accordance with the Helsinki convention on human rights. The ethics committee of CHU du Sart-Tilman and the university hospital legal department approved the project. The patients were informed about all the procedures and all signed the informed consent forms.

Data Availability. All data generated or analysed during this study are included in this published article/as supplementary information files.

Open Access. This article is licensed under a Creative Commons Attribution-NonCommercial 4.0 International License, which permits any non-commercial use, sharing, adaptation, distribution and reproduction in any medium or format, as long as you give appropriate credit to the original author(s) and the source, provide a link to the Creative Commons licence, and indicate if changes were made. The images or other third party material in this article are included in the article's
Creative Commons licence, unless indicated otherwise in a credit line to the material. If material is not included in the article's Creative Commons licence and your intended use is not permitted by statutory regulation or exceeds the permitted use, you will need to obtain permission directly from the copyright holder. To view a copy of this licence, visit http:// creativecommons.org/licenses/by-nc/4.0/.

\section{REFERENCES}

1. Belgian Cancer Registry group. Cancer in an ageing population: Belgium 2004-2016. Brussels: Belgian Cancer Registry; 2018. p. 65-74.

2. Belgian Cancer Registry group. Cancer incidence projections in Belgium 2015 to 2025. Brussels: Belgian Cancer Registry; 2017. p. 99-104.

3. Coates S, Kvedar J, Granstein R. Teledermatology: from historical perspective to emerging techniques of the modern era, part I: history, rationale and current practice. J Am Acad Dermatol. 2015;72(4): 563-74.

4. Coates S, Kvedar J, Granstein R. Teledermatology: from historical perspective to emerging techniques of the modern era, part II: emerging technologies in teledermatology, limitations and future directions. J Am Acad Dermatol. 2015;72(4):577-86.

5. Collins MK, Secrest A, Ferris L. Screening for melanoma. Melanoma Res. 2014;24(5):428-36.

6. Wolner $\mathrm{Z}$, Yélamos $\mathrm{O}$, Liopyris $\mathrm{K}$, Rogers $\mathrm{T}$, Marchetti M, Marghoob A. Enhancing skin cancer diagnosis with dermoscopy. Dermatol Clin. 2017;35(4):417-37.

7. Detrixhe A, Libon F, Mansuy M, et al. Melanoma masquerading as nonmelanocytic lesions. Melanoma Res. 2016;26(6):631-4.

8. Damsin T, Jacquemin P, Canivet G, Giet D, Gillet P, Nikkels AF. TeleSPOT project: early detection of melanoma by teledermoscopy in general practice. Rev Med Liege. 2019;74(12):650-4.

9. Vestergaard T, Pradad S, Schuster A, Laurinaviciene $\mathrm{R}$, Andersen MK, Bygum A. Diagnostic accuracy and interobserver concordance: teledermoscopy of 600 suspicious skin lesions in Southern Denmark. J Eur Acad Dermatol Venereol. 2020. https://doi.org/10. $1111 / j d v .16275$. 
10. Börve A, Dahlén Gyllencreutz J, Terstappen K, et al. Smartphone teledermoscopy referrals: a novel process for improved triage of skin cancer patients. Acta Derm Venereol. 2015;95(2):186-90.

11. Vestergaard T, Prasad S, Schuster A, et al. Introducing teledermoscopy of possible skin cancers in general practice in Southern Denmark. Fam Pract. 2020. https://doi.org/10.1093/fampra/cmaa041.

12. Reynolds C, Wyatt J. Open source, open standards, and health care information systems. J Med Internet Res. 2011. https://doi.org/10.2196/jmir.152.

13. Van Sinderen F, Tensen E, van der Heijden J, Witkamp L, Jaspers M, Peute L. Is teledermoscopy improving general practitioner skin cancer care? Stud Health Technol Inform. 2019;264:795-6.
14. Nikkels AF, Nikkels-Tassoudji N, Jerusalem-Noury E, et al. Skin cancer screening campaign in the german speaking community of Belgium. Acta Clin Belg. 2004;59(4):194-8.

15. Brochez L, Verhaeghe E, Bleyen L, Naeyaert JM. Diagnostic ability of general practitioners and dermatologists in discriminating pigmented skin lesions. J Am Acad Dermatol. 2001;44(6):979-86.

16. Gilling S, Mortz C, Vestergaard T. Patient satisfaction and expectations regarding mobile teledermoscopy in general practice for diagnosis of nonmelanoma skin cancer and malignant melanoma. Acta Derm Venereol. 2020. https://doi.org/10.2340/ 00015555-3. 